\title{
Decreased glucagon levels and decreased insulin secretion after sitagliptin versus mitiglinide administration with similar glycemic levels following an oral glucose load: a randomized crossover pharmaceutical mechanistic study
}

\author{
Yoshitaka Akiyama - Tomoko Morita-Ohkubo • Natsuko Oshitani • \\ Yuko Ohno • Yoshimasa Aso • Toshihiko Inukai • Masafumi Kakei • \\ Masanobu Kawakami - Takuya Awata - Shigehiro Katayama • Masafumi Matsuda \\ Received: 14 October 2014/ Accepted: 4 February 2015/Published online: 25 February 2015 \\ (C) The Japan Diabetes Society 2015
}

\begin{abstract}
Aims Both sitagliptin (SIT) and mitiglinide (MIT) can lower postprandial hyperglycemia. The purpose of this study was to examine differences in insulin and glucagon secretion after SIT or MIT administration when similar levels of plasma glucose (PG) were achieved for both agents following an oral glucose load.

Patients and methods We directly compared the effects of these two agents in 16 type-2 diabetic patients (M/ $\mathrm{F}=10 / 6$, age $66 \pm 3$ years old, HbA1c $6.6 \pm 0.5 \%$ ).
\end{abstract}

Y. Akiyama · T. Morita-Ohkubo $\cdot$ N. Oshitani ·

M. Matsuda $(\square)$

Department of Endocrinology and Diabetes, Saitama Medical

Center, Saitama Medical University, 1981 Kamoda,

Kawagoe-shi, Saitama, Japan

e-mail: matsudam-ind@umin.ac.jp

Y. Ohno

Central Laboratory, Saitama Medical Center, Saitama Medical

University, 1981 Kamoda, Kawagoe-shi, Saitama, Japan

Y. Aso

Department of Endocrinology and Metabolism, Dokkyo Medical University, 880 Kita-Kobayashi, Mibu, Shimotuga, Tochigi, Japan

T. Inukai

Department of Diabetes, Endocrinology and Hematology,

Dokkyo Medical University Koshigaya Hospital, 2-1-50

Minami-Koshigaya, Koshigaya, Saitama, Japan

M. Kakei · M. Kawakami

Department of Internal Medicine, Omiya Medical Center, Jichi

Medical School, Amanuma-Cho 1-847, Saitama, Japan

T. Awata $\cdot$ S. Katayama

Division of Endocrinology and Diabetes, Faculty of Medicine,

Saitama Medical University, 38 Morohongo, Moroyama,

Iruma-gun, Saitama, Japan
Patients received SIT (50 mg qd for 1 week and $100 \mathrm{mg}$ qd for an additional week) or MIT (10 mg tid for 2 weeks). After 2 weeks, patients crossed over to the other treatment. 75-g oral glucose tolerance tests were conducted before the study and after interventions.

Results The area under the curve (AUC) up to $180 \mathrm{~min}$ for the PG response was similar for both agents. While basal insulin secretion rates (ISR) were similar, incremental AUC of ISR was significantly lower in the SIT treatment $(522 \pm 108$ vs $702 \pm 288 \mathrm{pmol} / \mathrm{min} \mathrm{min}$, $p<0.01$ ), although the difference between the SIT and MIT treatments in the Matsuda index-which reflects insulin sensitivity-remained nonsignificant. Glucose-stimulated insulin secretion was similarly increased by the MIT and SIT treatments. Suppression of the AUC for glucagon was observed in the SIT treatment, while MIT treatment failed to suppress the glucagon concentration $(-432 \pm 2322$ vs MIT $1116 \pm 2520 \mathrm{pg} / \mathrm{ml} \mathrm{min}$, $p<0.05$ ). The basal proinsulin/insulin ratio was lower in the SIT treatment $(0.23 \pm 0.04$ vs MIT $0.26 \pm 0.36$, $p<0.05$ ).

Conclusions Although either SIT or MIT can be employed to reduce postprandial hyperglycemia, SIT induces changes in hormonal profiles that are more favorable to islet functions than MIT does.

Keywords Sitagliptin $\cdot$ Mitiglinide $\cdot$ Proinsulin

\section{Introduction}

The importance of avoiding postchallenge hyperglycemia to improve the quality of glycemic control in type-2 diabetic subjects has been underscored [1]. Oral hyperglycemic agents that reduce postprandial hyperglycemia 
include alpha-glucosidase inhibitors, glinides, and dipeptidyl peptidase-4 (DPP-4) inhibitors. While a beneficial effect of alpha-glucosidase inhibitors has been demonstrated [2], their efficacy is limited due to adverse events associated with their use and their low potential to reduce glucose levels, given that they are not capable of inducing insulin secretion. On the other hand, both glinides and DPP-4 inhibitors facilitate insulin secretion in pancreatic beta cells. Lowering the postprandial plasma glucose concentration minimizes damage to vessel walls, thus reducing nateglinide-induced atherosclerosis [3]. It has been postulated that DPP-4 inhibitors work against cardiovascular events, and the administration of these inhibitors has been demonstrated to result in improvements in surrogate markers [4]. Prospective studies examining their effects on cardiovascular endpoints are in progress.

Both DPP-4 inhibitors and glinides reduce the plasma glucose (PG) concentration after an oral glucose load by facilitating insulin secretion in pancreatic beta cells, albeit through different mechanisms. Sitagliptin is a DPP-4 inhibitor that inhibits the degradation of incretins, i.e., glucagon-like peptide-1 (GLP-1) and gastric inhibitory polypeptide (GIP) [5]. GLP-1 acts through its receptors on pancreatic beta cells by enhancing insulin secretion during the hyperglycemic state while also normalizing the glucagon response to glycemic states, while GIP works to enhance glucagon secretion [6]. However, glinides act through the sulfonylurea receptor (SUR)-1 of pancreatic beta cells, inducing insulin secretion [7]. Mitiglinide is a synthesized non-sulfonylurea hypoglycemic agent that exerts a rapid but short-lasting hypoglycemic effect. This compound has been reported to enhance glucagon secretion transiently following sustained suppression [8].

Therefore, the responses of insulin and glucagon to an oral glucose load following the administration of sitagliptin or mitiglinide are of great interest. However, there is no published report of a direct comparison of those two agents with regards to islet function, especially when similar levels of plasma glucose are achieved. In the work described in the present paper, we conducted a randomized crossover study to find differences in the effects of these two agents on hormonal levels following an oral glucose load when similar hypoglycemic potency was anticipated.

\section{Patients and methods}

Study design and participants

This randomized crossover multicenter study was conducted to examine the effects of sitagliptin (a DPP-4 inhibitor) and mitiglinide (a glinide compound) on insulin secretion and insulin sensitivity (possibly mediated by
GLP-1 through its action on alpha cells as well as beta cells in the pancreas) during oral glucose tolerance tests (OGTTs). The main outcome was the area under the curve (AUC) for glucagon response during the OGTT following sitagliptin treatment or mitiglinide treatment.

Subjects had to be at least 20 years old and less than 75 years old. They also had to be type- 2 diabetic patients with stable glycemic control for more than two months, and their HbA1c was required to be $<9.4 \%$. Subjects taking pioglitazone or metformin were included. Subjects undergoing treatment with insulin, type-1 diabetic subjects, subjects taking alpha-glucosidase inhibitors or sulfonylureas, subjects with nephrotic syndrome, and subjects taking steroids, immune suppression medication, or azole antifungal medication were excluded. Subjects taking HIV protease inhibitors, subjects who had previously suffered serious adverse events while taking DPP-4 inhibitors and/ or glinides, subjects who had a stroke or acute coronary syndrome within 6 months before enrollment, subjects with severe heart failure (NYHA class 3 or higher) or severe arrhythmia, subjects who had elevated liver enzymes, subjects with malignancy, subjects who were pregnant or were attempting to get pregnant, and subjects with other conditions that led the doctor in charge to decide that they were not eligible for this study were also excluded.

Informed consent was obtained before the study. The original protocol and consent form was approved by the ethics committee of Saitama Medical Center, Saitama Medical University, and the protocol and consent form for each institute was approved by local independent ethics committees or institutional review boards before trial initiation.

\section{Procedures}

After obtaining written consent, the order in which sitagliptin and mitiglinide were administered to eligible patients was randomized using a central web response system. Patients received sitagliptin (50 mg qd for 1 week and $100 \mathrm{mg}$ qd for an additional week) or mitiglinide (10 mg tid for 2 weeks) according to the randomization. These doses of both agents were designed to achieve similar glucose-lowering effects, based on the information in the Japan Pharmaceutical Reference (http://www.esearch.ne.jp/ jpr/). After two weeks, each patient crossed over to the other treatment. 75-g OGTTs were conducted before the study and after interventions. A flow diagram of the study is depicted in Fig. 1.

An OGTT was started after a 10-12 h overnight fasting period at 9 am after basal sampling of blood for glycoalbumin and HbAlc as well as substrates and hormones, as described below. After basal sampling, patients were asked 
Fig. 1 Flow diagram of the study. During week 1, $50 \mathrm{mg}$ sitagliptin were administered orally in the morning after breakfast; in week 2 , this was increased to $100 \mathrm{mg}$ sitagliptin. Mitiglinide treatment consisted of $10 \mathrm{mg}$ mitiglinide tid orally before each meal for two weeks. A 75-g OGTT was performed at the end of each treatment period

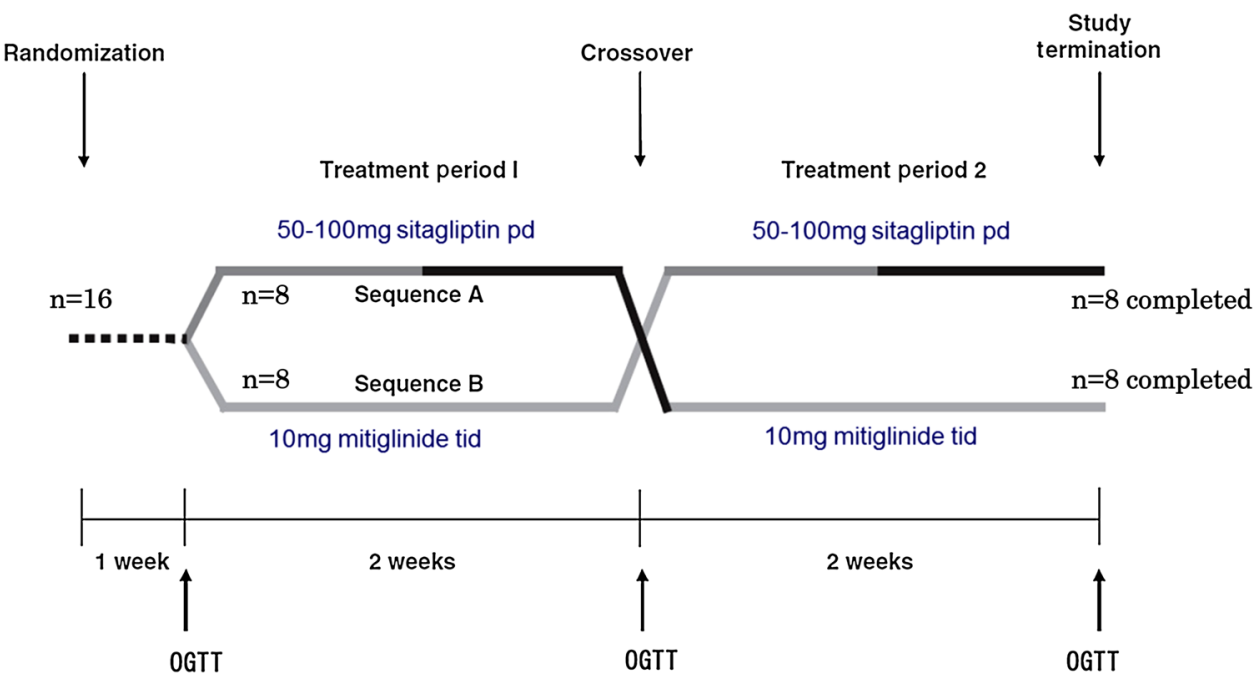

to take mitiglinide $10 \mathrm{mg}$, when assigned, before the administration of an oral glucose load. A stopwatch was started when the thyroid cartilage moved down after the first swallow of Trelan ${ }^{\circledR}-$ G75 (Ajinomoto Pharmaceuticals Co. Ltd., Tokyo, Japan), which is equivalent to $75 \mathrm{~g}$ glucose as a metabolic product. Alternatively, patients were asked to take sitagliptin they had finished drinking the Trelan ${ }^{\circledR}-$ G75. Blood was sampled at 30,60, 90, 120, and 180 min after the start of the test in order to measure glucose, triglyceride, insulin, C-peptide, proinsulin, glucagon, and GLP-1.

Plasma or serum samples were obtained according to the recommended procedure for assays. The samples were cooled at $4{ }^{\circ} \mathrm{C}$ before separation to obtain serum or plasma. They were then frozen at $-80{ }^{\circ} \mathrm{C}$ before they were sent to the central laboratory of Mitsubishi Kagaku Bio-Clinical Laboratories, Inc. (Tokyo, Japan). Glucose and triglyceride were measured enzymatically, and insulin, C-peptide, proinsulin, glucagon, and GLP-1 were measured by ELISA using specific antibodies in the central laboratory. Samples obtained for the glucagon assay were placed into vials containing aprotinin. Samples were extracted for the GLP1 assay and added to vials containing diprotin A (a DPP-4 inhibitor) and GLP-1 before measurements. Biologically active forms of GLP-1 [i.e., GLP-1(7-36)amide and GLP1(7-37)] were measured by ELISA (Linco Research, St. Louis, MO, USA) according to the Guideline for Incretin Measurement (http://www.jds.or.jp/uploads/photos/786. pdf) issued by the Japan Diabetes Society.

\section{Calculation and statistical analysis}

The insulin secretion rate (ISR) during the OGTT was calculated by deconvolving the plasma $\mathrm{C}$-peptide concentration using the ISEC software package developed by Hovorka et al. [9]. The ISR was related to the glucose stimulus by dividing the incremental area under the ISR curve by the incremental area under the plasma glucose curve. HOMA-IR and HOMA-beta were calculated as described previously [10]. The Matsuda index incorporates both hepatic and muscle components of insulin sensitivity, correlates well with the results of the euglycemic insulin clamp technique, and was calculated as reported previously [11]. This made it easier to understand values, since the simple raw value obtained from the calculation was too large and difficult to assess. The deposition index (insulin secretion/insulin resistance) was determined by dividing $\Delta \mathrm{ISR} / \Delta \mathrm{PG}$ by the severity of insulin resistance (IR) $[\Delta \mathrm{ISR}(\mathrm{AUC}) / \Delta \mathrm{G}(\mathrm{AUC})$ divided by $\mathrm{IR}]$, where IR was measured as the inverse of the Matsuda index [12]. The incremental area under the concentration curve of hormone or substrate concentration was calculated according the trapezoid rule.

The Wilcoxon signed-rank test (Mann-Whitney $U$ test) was used to compare the data obtained from the OGTTs. All statistical analyses were performed using SPSS version 21.0 (IBM SPSS Statistics, Chicago, IL, USA). Data are expressed as the mean \pm SD. Data with $p$ values of $<0.05$ were considered significant.

\section{Results}

Sixteen type-2 diabetic patients were randomized to start either medication in addition to the pills that they were already taking. We directly compared the effects of these two agents in the 16 type- 2 diabetic patients, as shown in Table 1. Average age was $66.2 \pm 3.4$ years old, and average BMI was $24.4 \pm 3.6 \mathrm{~kg} / \mathrm{m}^{2}$. Average duration of diabetes was $11.5 \pm 9.1$ years, and average recent $\mathrm{HbA1c}$ was $6.6 \pm 0.5 \%$. No advanced diabetic complications were reported. There were no differences between the 
features of the subjects who started mitiglinide first and those of the subjects who started sitagliptin first. Subjects taking pioglitazone $(n=2)$ and metformin $(n=9)$ were

Table 1 Baseline characteristics of subjects

\begin{tabular}{llll}
\hline & Total & $\begin{array}{l}\text { Mitiglinide } \\
\text { treatment }\end{array}$ & $\begin{array}{l}\text { Sitagliptin } \\
\text { treatment }\end{array}$ \\
\hline$n(\mathrm{M} / \mathrm{F})$ & $16(10 / 6)$ & $8(4 / 4)$ & $8(6 / 2)$ \\
Age (years old) & $66.2 \pm 3.4$ & $66.1 \pm 3.9$ & $66.3 \pm 3.2$ \\
BMI $\left(\mathrm{kg} / \mathrm{m}^{2}\right)$ & $24.4 \pm 3.6$ & $25.0 \pm 4.4$ & $23.7 \pm 2.6$ \\
Duration of diabetes & $11.5 \pm 9.1$ & $14.5 \pm 12.0$ & $8.5 \pm 3.7$ \\
$\quad($ years $)$ & $6.6 \pm 0.5$ & $6.5 \pm 0.4$ & $6.7 \pm 0.7$ \\
HbAlc $(\%)$ & $116 \pm 27$ & $113 \pm 27$ & $118 \pm 28$ \\
FPG (mg/dl) & $94 \pm 29$ & $81 \pm 16$ & $107 \pm 34$ \\
LDL-chol (mg/dl) & $65 \pm 19$ & $64 \pm 23$ & $66 \pm 17$ \\
HDL-chol (mg/dl) & $110 \pm 65$ & $116 \pm 86$ & $103 \pm 37$ \\
TG (mg/dl) & $0.82 \pm 0.21$ & $0.87 \pm 0.19$ & $0.78 \pm 0.23$ \\
Creatinine (mg/dl) & & & \\
\hline
\end{tabular}

Data are expressed as the mean $\pm \mathrm{SD}$ asked to continue taking them as prescribed during the whole study. The area under the curve (AUC) up to 180 min for PG response was similar regardless of the agent taken, and lower than before treatment (Table 2). The basal insulin secretion rates $[0.86 \pm 0.15$ at baseline (BASE), $1.03 \pm 0.38$ after mitiglinide (MIT) treatment, and $1.11 \pm 0.56 \mathrm{pmol} / \mathrm{kg}$ per min after sitagliptin (SIT) treatment] were similar before the study, and there were no differences after taking mitiglinide or sitagliptin.

As expected, an increase in GLP-1 was only detected in the sitagliptin treatment, as shown in Fig. 2. The basal GLP-1 level was three times higher $(3.53 \pm 0.96 \mathrm{pmol} / \mathrm{l})$ after sitagliptin treatment than those of BASE $(1.14 \pm 0.32)$ and MIT $(1.37 \pm 0.69)$.

Excursion curves for glucose, insulin, C-peptide, proinsulin, glucagon, and triglyceride during 75-g OGTTs in the subjects before and two weeks after the start of mitiglinide or sitagliptin intervention are depicted in Fig. 3. To facilitate a comparison of the substrate and hormone responses, we calculated the area under the curve in each
Table 2 Summary of parameters during OGTTs

OGTTs were conducted before (BASE) and 2 weeks after the start of intervention with mitiglinide and sitagliptin. Before calculating ratios, the units for insulin, C-peptide, and proinsulin were converted to $\mathrm{pmol} / \mathrm{l}, \mathrm{nmol} / \mathrm{l}$, and $\mathrm{pmol} / \mathrm{l}$, respectively. The AUC was calculated up to $180 \mathrm{~min}$. Data are expressed as the mean \pm SD of 16 subjects. Statistics were examined using the Wilcoxon rank-sum test $* p<0.05$, ** $p<0.01 \mathrm{vs}$ mitiglinide

\begin{tabular}{|c|c|c|c|}
\hline & BASE & Mitiglinide & Sitagliptin \\
\hline \multicolumn{4}{|l|}{ Basal } \\
\hline GLP-1 (pmol/L) & $1.14 \pm 0.32$ & $1.37 \pm 0.69$ & $3.53 \pm 0.96^{* *}$ \\
\hline FPG (mg/dl) & $115.5 \pm 26.5$ & $118.9 \pm 32.6$ & $107.9 \pm 4.9 * *$ \\
\hline Glycoalbumin (\%) & $18.0 \pm 1.8$ & $17.8 \pm 1.9$ & $17.7 \pm 0.6$ \\
\hline Glucagon (pg/ml) & $73.9 \pm 19.9$ & $70.8 \pm 18.1$ & $76.6 \pm 4.3$ \\
\hline Insulin $(\mu \mathrm{U} / \mathrm{ml})$ & $3.7 \pm 2.1$ & $4.2 \pm 3.4$ & $4.7 \pm 1.2$ \\
\hline C-peptide (ng/ml) & $0.95 \pm 0.20$ & $1.16 \pm 0.57$ & $1.26 \pm 0.20$ \\
\hline Proinsulin $(\mathrm{pmol} / \mathrm{L})$ & $5.7 \pm 3.2$ & $6.2 \pm 2.3$ & $5.3 \pm 0.6^{*}$ \\
\hline TG $(\mathrm{mg} / \mathrm{dl})$ & $110 \pm 65$ & $92 \pm 47$ & $112 \pm 14 * *$ \\
\hline LDL-chol (mg/dl) & $93.8 \pm 29.3$ & $92.8 \pm 23.4$ & $88.6 \pm 5.2$ \\
\hline HDL-chol (mg/dl) & $64.7 \pm 19.4$ & $65.3 \pm 17.2$ & $63.4 \pm 4.8$ \\
\hline Proinsulin/insulin & $0.26 \pm 0.15$ & $0.26 \pm 0.13$ & $0.23 \pm 0.04^{*}$ \\
\hline Proinsulin/C-peptide & $21.7 \pm 11.7$ & $20.7 \pm 8.6$ & $17.4 \pm 8.4^{* *}$ \\
\hline HOMA-IR & $1.1 \pm 0.8$ & $1.4 \pm 1.5$ & $1.4 \pm 1.6$ \\
\hline НОМА- $\beta$ & $30.0 \pm 21.9$ & $29.3 \pm 15.9$ & $40.7 \pm 32.9$ \\
\hline Basal ISR (pmol/kg per min) & $0.86 \pm 0.15$ & $1.03 \pm 0.38$ & $1.11 \pm 0.56$ \\
\hline \multicolumn{4}{|l|}{ OGTT } \\
\hline 2 h PG & $240 \pm 88$ & $187 \pm 77$ & $180 \pm 75$ \\
\hline 3 h PG & $184 \pm 92$ & $134 \pm 85$ & $134 \pm 66$ \\
\hline$\triangle \mathrm{AUC}$ of GLP-1 & $756 \pm 749$ & $837 \pm 934$ & $2056 \pm 2499 * *$ \\
\hline$\Delta \mathrm{AUC}$ of glucose & $19135 \pm 7382$ & $10771 \pm 6809$ & $11903 \pm 7531$ \\
\hline$\Delta \mathrm{AUC}$ of insulin & $2258 \pm 1476$ & $3244 \pm 2069$ & $2556 \pm 2086^{*}$ \\
\hline$\triangle \mathrm{AUC}$ of glucagon & $-129 \pm 2693$ & $1110 \pm 2513$ & $-428 \pm 2330^{*}$ \\
\hline$\triangle \mathrm{AUC}$ of C-peptide & $455 \pm 204$ & $628 \pm 287$ & $477 \pm 228 * *$ \\
\hline$\Delta \mathrm{AUC}$ of proinsulin & $2077 \pm 1165$ & $2747 \pm 1616$ & $1803 \pm 1182 * *$ \\
\hline$\triangle \mathrm{AUC}$ of $\mathrm{TG}$ & $-2080 \pm 4198$ & $-953 \pm 1209$ & $-2379 \pm 3065$ \\
\hline$\triangle \mathrm{AUC}$ of ISR & $508 \pm 214$ & $693 \pm 283$ & $527 \pm 101 * *$ \\
\hline Matsuda index (MI) & $11.0 \pm 6.5$ & $9.9 \pm 5.2$ & $11.1 \pm 6.6$ \\
\hline$\triangle \mathrm{AUC}$ of ISR/AAUC of glucose $\times \mathrm{MI}$ & $0.39 \pm 0.44$ & $1.16 \pm 2.46$ & $0.79 \pm 0.81$ \\
\hline
\end{tabular}


case (AUC). The AUC for glucagon response during the OGTT after sitagliptin treatment was not significantly decreased compared to the baseline $(76.9 \pm 18.9$ in MIT, $74.2 \pm 22.4$ in SIT, and $73.2 \pm 19.9 \mathrm{ng} / \mathrm{ml}$ in BASE). Despite a slight, nonsignificant increase in basal glucagon levels in the SIT treatment ( $76.6 \pm 4.3$ vs $70.8 \pm 18.1$ in MIT, and $73.9 \pm 19.9 \mathrm{pg} / \mathrm{ml}$ in BASE), a significant decrease in the AUC of glucagon was observed, whereas MIT treatment failed to suppress the glucagon concentration $(-432 \pm 2322$ vs MIT $1116 \pm 2520 \mathrm{pg} / \mathrm{ml} \mathrm{min}$, $p<0.05$ ) (Fig. 4).

The SIT and MIT treatments did not yield significantly different values of HOMA-IR or HOMA-beta. There also were no differences between the treatments in the Matsuda index $(11.1 \pm 6.6$ in SIT, $9.9 \pm 5.2$ in MIT $)$.

While basal insulin secretion rates (ISRs) were similar for both treatments, the incremental AUC of ISR was significantly lower in the SIT treatment $(522 \pm 108$ vs $702 \pm 288 \mathrm{pmol} / \mathrm{kg}$ per min min, $p<0.01)$, and the $\triangle$ AUC of insulin $(2556 \pm 2088$ vs $3240 \pm 2070 \mu \mathrm{U} /$ $\mathrm{ml}$ min in MIT, $p<0.05)$ and the $\Delta$ AUC of C-peptide $(486 \pm 234$ vs $630 \pm 288 \mathrm{ng} / \mathrm{ml} \mathrm{min}$ in MIT, $p<0.01)$ were smaller in the SIT treatment. There was no difference between the treatments in the disposition index (Table 2). Figure 5a plots the ISR versus time. This excursion curve indicates that mitiglinide treatment increased the ISR as compared with the ISR afforded by sitagliptin treatment or the baseline rate. Plotting the ISR data against the concomitant plasma glucose concentrations measured during the OGTT enabled the glucose-stimulated insulin secretion (GSIS) to be assessed (Fig. 5b). The GSIS was not decreased by sitagliptin administration.

The AUC of proinsulin was decreased in the SIT treatment $(2700 \pm 684$, vs MIT $3852 \pm 1764, p<0.01$; vs CON $3096 \pm 1548 \mathrm{pmol} / \mathrm{l} \mathrm{min}, p<0.05)$. Basal triglyceride levels were reduced by MIT treatment ( $92 \pm 47$ vs $112 \pm 14 \mathrm{mg} / \mathrm{dl}$ in SIT, $p<0.01)$. Although this decrease was not significant, the $\triangle \mathrm{AUC}$ of $\mathrm{TG}$ was lower $(-2376 \pm 3060$ vs $-954 \pm 1206 \mathrm{mg} / \mathrm{dl} \mathrm{min}$ in MIT $)$.

There were no differences in the values of parameters for subjects who were treated with sitagliptin first and those treated with mitiglinide first in this study. There were no treatment-related abnormalities based on laboratory measurements such as liver function tests, creatinine, and urinalysis for ketones and proteins. Furthermore, there were no changes in vital signs such as heart rate and blood pressure with either treatment. There were no hypoglycemic episodes.

\section{Discussion}

Similar glycemic levels were achieved after an oral glucose load in this study after treatment with either sitagliptin or

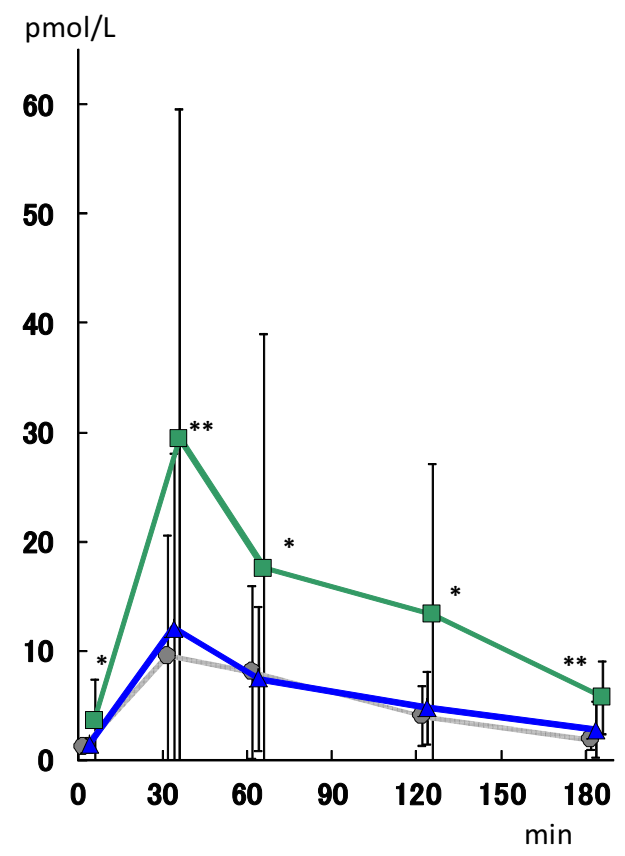

Fig. 2 Plasma GLP-1 concentrations after 75-g OGTTs performed before (gray circles) and two weeks after the start of mitiglinide treatment (blue triangles) or two weeks after the start of sitagliptin treatment (green squares). Each data value is expressed as the mean $\pm \mathrm{SD}$. Statistics were examined using the Wilcoxon rank-sum test. $* p<0.01, * * p<0.001$ vs mitiglinide

mitiglinide. Although the area under the curve (AUC) for glucagon response during an OGTT was slightly lower for the sitagliptin treatment, it was not significantly less than that seen with the mitiglinide treatment. However, a decrease in the AUC for glucagon was observed after treatment with sitagliptin, while treatment with mitiglinide failed to suppress glucagon concentration. There was no difference between the treatments in insulin resistance and insulin sensitivity as assessed by HOMA-IR and the Matsuda index. Although at first glance the ISR seemed to be greater for the mitiglinide treatment, the disposition indices were similar for both treatments. In addition, the GSIS was not lower in patients treated with sitagliptin. Since this study was designed to achieve similar hypoglycemic potencies with the two medications, there were no differences between the treatments in glycoalbumin and 2-h PG after OGTTs. However, the FPG was lower when sitagliptin was assigned, as the short duration of action of mitiglinide meant that it was not potent enough to counter glucose toxicity over consecutive days. On the other hand, it seems likely that sitagliptin continued to work until at least the next morning when the dose was $100 \mathrm{mg}$ per day. Sitagliptin at $100 \mathrm{mg}$ per day may significantly suppress DPP-4 overnight, as demonstrated by a dose-response increase in inhibition [5] It is therefore no wonder that GLP-1 levels were increased during the fasting state, resulting in a reduced fasting PG. 
Fig. 3 Excursion curves for a glucose, $\mathbf{b}$ insulin, $\mathbf{c} \mathrm{C}$ peptide, $\mathbf{d}$ proinsulin,

e glucagon, and $\mathbf{f}$ triglyceride during 75-g OGTTs in subjects before (gray circles) and two weeks after the start of mitiglinide treatment (blue triangles) or two weeks after the start of sitagliptin treatment (green squares). Each data value is expressed as the mean \pm SD. Statistics were examined using the Wilcoxon rank-sum test. $* p<0.05$, $* * p<0.01$ vs mitiglinide
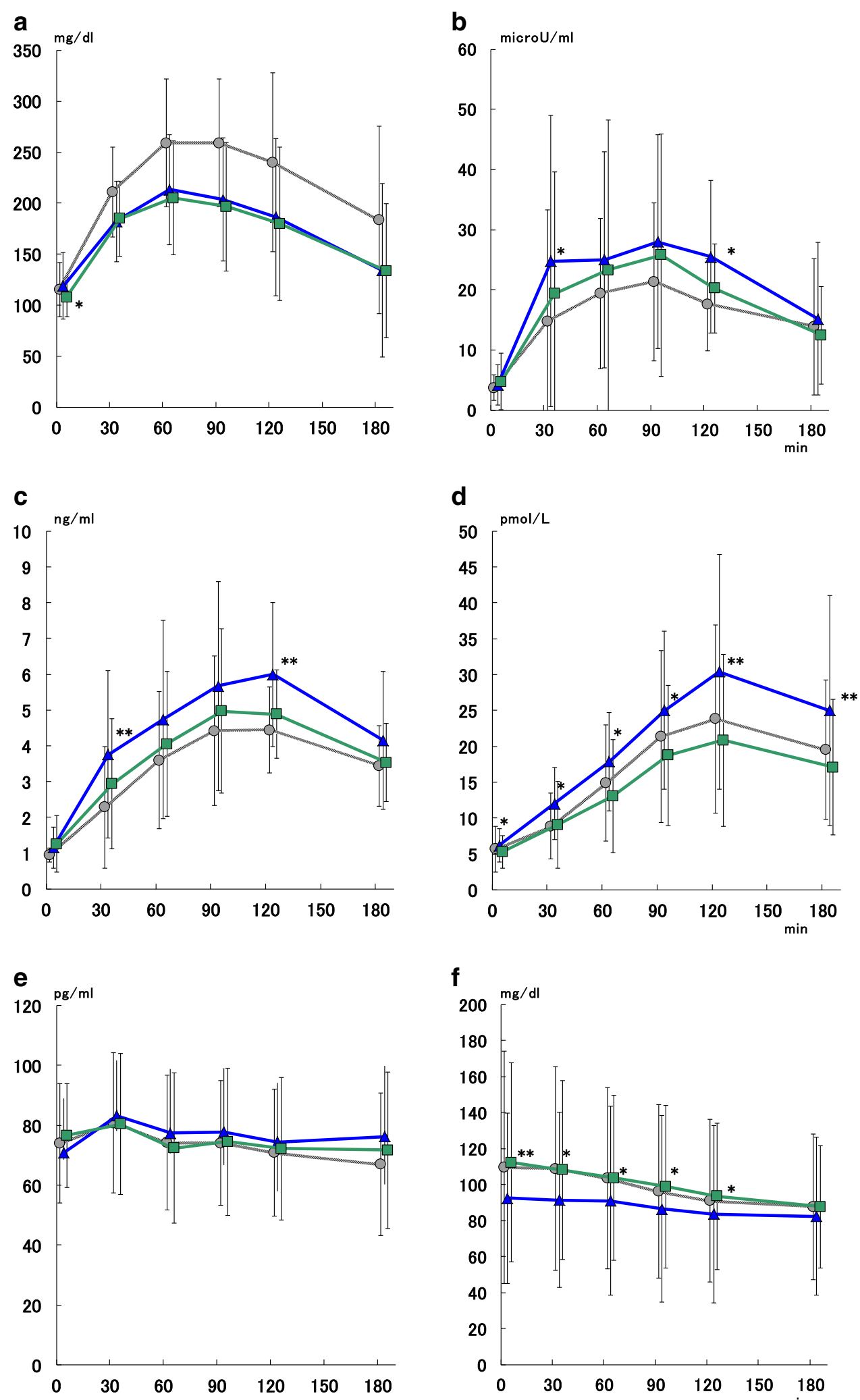

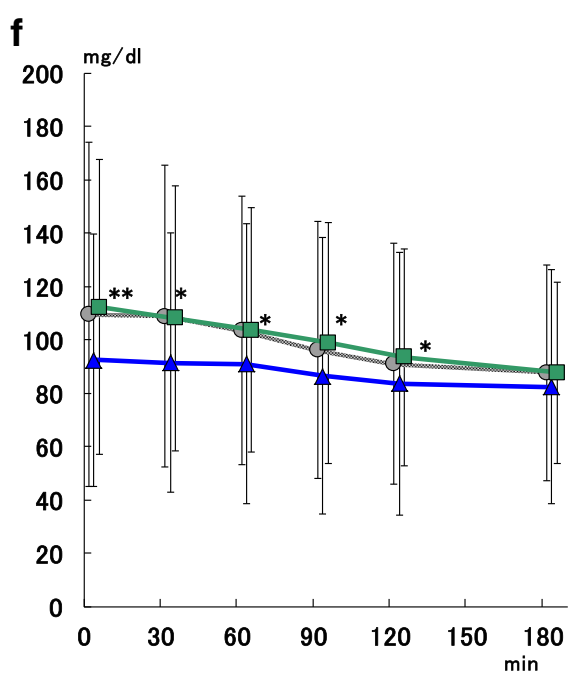

This may have caused the slight increase in the basal GSIS in the sitagliptin group compared with the baseline GSIS and that observed in the mitiglinide group (Fig. 4).
Although both sitagliptin and mitiglinide facilitated insulin secretion, reduced glucagon responses and reduced insulin responses were observed in the sitagliptin treatment 


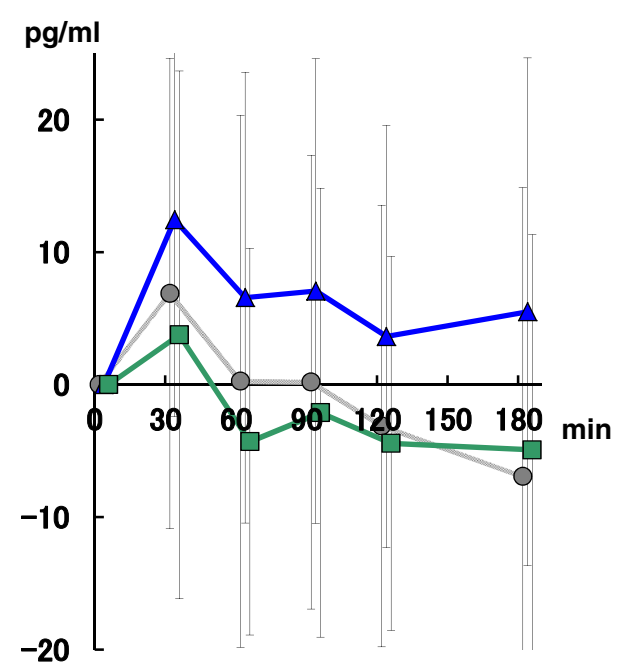

Fig. 4 Excursion curves showing the AUC for glucagon during 75-g OGTTs in subjects before (gray circles) and two weeks after the start of mitiglinide treatment (blue triangles) or two weeks after the start of sitagliptin treatment (green squares). Each data value is expressed as the mean (bold horizontal bar) $\pm \mathrm{SD}$ (vertical bar)

compared to those seen in the mitiglinide treatment. In addition, levels of proinsulin/insulin and proinsulin/Cpeptide (indicators of beta-cell working stress) were significantly lower with sitagliptin treatment.

The D-phenylalanine derivative nateglinide, a short-acting insulin secretagogue, has been reported to have DPP-4-inhibitor-like potency [13] or the ability to secrete GLP-1 through its amino-acid-like structure independent of a K-ATP channel [14]. However, mitiglinide has never been reported to act in this way, and we did not observe any additional increases in GLP-1 concentration after an oral glucose load during mitiglinide intervention (Fig. 2). Comparisons of the effects of a DPP-4 inhibitor and those of mitiglinide have been conducted in mice [15] and in humans [16]. Repaglinide also had no effect on GLP-1 in humans [17].

We also observed a significant acute decrease in the triglyceride concentration after glucose administration with sitagliptin treatment. In a previous study, when postprandial levels of both lipids and glucagon after three months of treatment with sitagliptin were compared with those after three months of treatment with nateglinide, triglycerides were found to be significantly suppressed in the sitagliptin group [7]. A significant decrease in the serum glucagon level was also observed at $1 \mathrm{~h}$ postprandially in the sitagliptin group in that study. Although the duration of treatment was different in our study, similar responses in terms of triglycerides and glucagon were observed. However, in our study, the fasting triglyceride and glucagon levels showed the opposite behavior after the two weeks' intervention. Since the fasting PG was significantly lower after sitagliptin treatment due to increased fasting insulin a

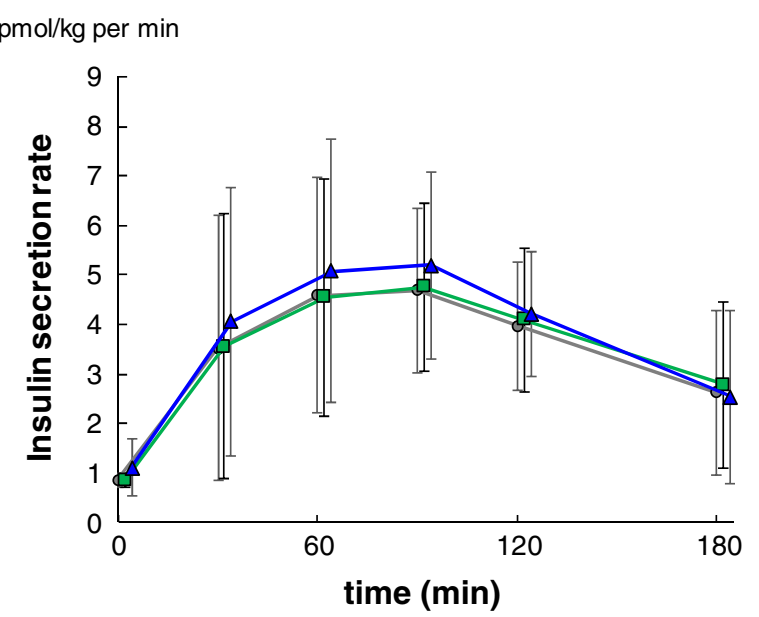

b

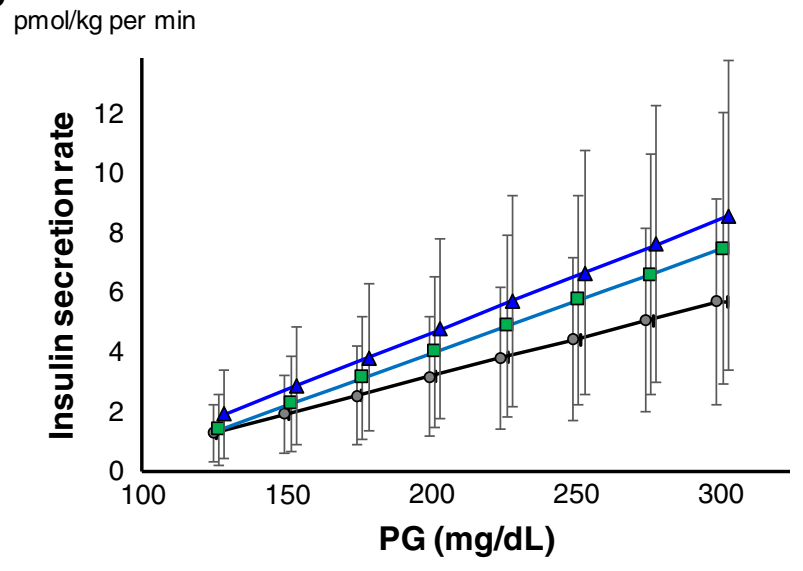

Fig. 5 a The insulin secretion rate in subjects was calculated and plotted versus time during a 75-g OGTT performed before (gray circles) and two weeks after the start of mitiglinide treatment (blue triangles) or two weeks after the start of sitagliptin treatment (green squares). b Plotting the ISR data against the concomitant plasma glucose concentrations measured during the OGTT enabled the glucose-stimulated insulin secretion (GSIS) to be assessed in subjects before (gray circles) and two weeks after the start of mitiglinide treatment (blue triangles) or two weeks after the start of sitagliptin treatment (green squares). Each data value is expressed as the mean (bold horizontal bar) $\pm \mathrm{SD}$ (vertical bar)

concentrations and GLP-1 levels, glucagon secretion may be controlled and suppressed using this intervention, as previously proposed [18]. It is possible that glucagon levels were slightly elevated to avoid hypoglycemia, resulting in a slight increase in fasting triglycerides, which may help to avoid hypoglycemic attacks while using sitagliptin.

We believe that using the plasma C-peptide concentration as a measure of pre-hepatic insulin secretion provides a more direct measure of GSIS and beta-cell function, although another study [7, 19] suggested that hepatic insulin extraction does not affect insulin-derived calculations of beta-cell function. We conducted this study by administering an oral glucose load, since excluding the 
gastrointestinal tract by administering the glucose load intravenously may limit our physiologic understanding of beta-cell function in vivo.

This study was primarily designed to compare the acute glycemic responses of the patients to the two treatments, so a longer-term study was not considered necessary. Longterm use of these agents may yield different results, but they would be affected by any changes in body weight or other factors. However, for the first month of SIT treatment, the study reported in a previous study [7] gave similar results to those seen for the SIT treatment in our study, while the effect of nateglinide was limited in the same study. Although nateglinide exhibits DPP-4-inhibitorlike action [13, 14, 20], its beneficial effects were significantly exceeded by those of sitagliptin. In addition, nateglinide failed to show any beneficial effects on glucose metabolism and cardiovascular events in the NAVIGATOR study [21]. Possible reasons include increased body weight and the occurrence of hypoglycemia, which are not likely to be induced by sitagliptin.

One limitation of the present study was the lack of a medication washout period between the two treatment periods. However, since the half-lives of both sitagliptin (12 h) and mitiglinide $(1.2 \mathrm{~h})$ are short, and assessments were conducted two weeks after the patients had crossed over to the other treatment, any carryover effect is unlikely. A test for carryover using patients nested within sequence as the error term revealed no evidence of any carryover effect. Moreover, as the study was primarily designed to compare the acute glycemic responses of the patients to the two treatments, a longer study duration was not considered necessary; this approach was also adopted in a similar study [22]. In addition, terminating the administration of a hypoglycemic agent may cause glucose toxicity, which may affect the results. However, since the order of agent administration was randomized for the patients, the conclusions drawn based on the results of this study are scientifically robust.

There are other limitations to this study. The number of subjects included was not large. However, it should be possible to compare the responses of each patient to the two treatments in this study design, and all of the participants returned at the exact time scheduled for this study. Therefore, we believe that the results reflect basic pharmacological data for humans, and increasing the number of subjects may not be ethically appropriate. Also, we only administered an oral glucose load; we did not use mixed meals. However, we wanted to elucidate differences in the mechanisms of action of sitagliptin and mitiglinide, especially on insulin secretion and insulin sensitivity (possibly mediated by GLP-1 through its action on alpha cells as well as beta cells in the pancreas) during OGTTs. The data shown here should prove helpful when attempting to understand differences in the effects of DPP-4 inhibitors and glinides when they are used in clinical situations.

In conclusion, the glucagon and insulin responses were suppressed by sitagliptin treatment as compared to mitiglinide treatment after an oral glucose load. In addition, as demonstrated by reduced proinsulin levels, less beta-cell stress occurred in the sitagliptin treatment. Both the normalization of alpha-cell function and the protection of betacell function in the pancreatic islets by sitagliptin were successfully demonstrated in this study. It may be better to use DPP-4 inhibitors rather than mitiglinide when postprandial hyperglycemia is a concern in the treatment of type-2 diabetic patients.

Acknowledgments This work was planned and conducted by the Saitama Incretin Study Group, and was funded via a grant from the Waksman Foundation of Japan, Inc. The authors are members of the Saitama Incretin Study Group, of which Shigehiro Katayama is the president. The number of this clinical trial in the UMIN clinical trial registration system is UMIN000005283. The UMIN Registry has been officially accepted by the ICMJE (International Committee of Medical Journal Editors). Part of the data obtained in this study was presented at the annual meeting of the American Diabetes Association in 2012, and the calculations of insulin secretion rates were presented as an abstract at the annual meeting of the Japan Diabetes Society in 2013.

Conflict of interest Yoshimasa Aso received lecture fees from Nippon Boehringer Ingelheim Co., Ltd., Eli Lilly Japan K.K., and MDS K.K. Shigeharu Katayama received research grants from MSD K.K. and Daiichi Sankyo Pharma Ltd. Yoshitaka Akiyama, Tomoko Morita-Okubo, Natsuko Oshitani, Yuko Ohno, Toshihiko Inukai, Kakei Masafumi, Kawakami Masanobu, Takuya Awata, and Masafumi Matsuda declare that they have no conflict of interest.

Human rights statement and informed consent All procedures followed were in accordance with the ethical standards of the responsible committee on human experimentation (institutional and national) and with the Helsinki Declaration of 1964 and its subsequent revision. Informed consent or a substitute for it was obtained from all patients before they were included in the study.

\section{References}

1. DECODE Study Group, European Diabetes Epidemiology Group. Glucose tolerance and mortality: comparison of WHO and American Diabetes Association diagnostic criteria. Diabetes Epidemiology: Collaborative analysis Of Diagnostic criteria in Europe. Lancet. 1999;354:617-621.

2. Chiasson JL, Josse RG, Gomis R, Hanefeld M, Karasik A, Laakso M. Acarbose treatment and the risk of cardiovascular disease and hypertension in patients with impaired glucose tolerance: the STOP-NIDDM trial. JAMA. 2003;290:486-94.

3. Mita T, Watada H, Shimizu T, Tamura Y, Sato F, Watanabe T, et al. Nateglinide reduces carotid intima-media thickening in type 2 diabetic patients under good glycemic control. Arterioscler Thromb Vasc Biol. 2007;27:2456-62.

4. Chrysant SG, Chrysant GS. Clinical implications of cardiovascular preventing pleiotropic effects of dipeptidyl peptidase-4 inhibitors. Am J Cardiol. 2012;109:1681-5. 
5. Herman GA, Bergman A, Stevens C, Kotey P, Yi B, Zhao P, et al. Effect of single oral doses of sitagliptin, a dipeptidyl peptidase-4 inhibitor, on incretin and plasma glucose levels after an oral glucose tolerance test in patients with type 2 diabetes. J Clin Endocrinol Metab. 2006;91:4612-9.

6. Meier JJ, Gallwitz B, Siepmann N, Holst JJ, Deacon CF, Schmidt WE, et al. Gastric inhibitory polypeptide (GIP) dose-dependently stimulates glucagon secretion in healthy human subjects at euglycaemia. Diabetologia. 2003;46:798-801.

7. Kojima Y, Kaga H, Hayashi S, Kitazawa T, Iimura Y, Ohno M, et al. Comparison between sitagliptin and nateglinide on postprandial lipid levels: the STANDARD study. World J Diabetes. 2013;4:8-13.

8. Kinukawa M, Ohnota H, Ajisawa Y. Effect of a non-sulphonylurea hypoglycaemic agent, KAD-1229 on hormone secretion in the isolated perfused pancreas of the rat. $\mathrm{Br} \mathrm{J}$ Pharmacol. 1996;117:1702-6.

9. Hovorka R, Soons PA, Young MA. ISEC: a program to calculate insulin secretion. Comput Methods Programs Biomed. 1996;50: 253-64.

10. Matthews DR, Hosker JP, Rudenski AS, Naylor BA, Treacher DF, Turner RC. Homeostasis model assessment: insulin resistance and beta-cell function from fasting plasma glucose and insulin concentrations in man. Diabetologia. 1985;28:412-9.

11. Matsuda M, DeFronzo RA. Insulin sensitivity indices obtained from oral glucose tolerance testing: comparison with the euglycemic insulin clamp. Diabetes Care. 1999;22:1462-70.

12. Abdul-Ghani MA, Matsuda M, Jani R, Jenkinson CP, Coletta $\mathrm{DK}, \mathrm{Kaku} \mathrm{K}$, et al. The relationship between fasting hyperglycemia and insulin secretion in subjects with normal or impaired glucose tolerance. Am J Physiol Endocrinol Metab. 2008;295:E401-6.

13. Duffy NA, Green BD, Irwin N, Gault VA, McKillop AM, O'Harte FP, et al. Effects of antidiabetic drugs on dipeptidyl peptidase IV activity: nateglinide is an inhibitor of DPP IV and augments the antidiabetic activity of glucagon-like peptide-1. Eur J Pharmacol. 2007;568:278-86.

14. Kitahara Y, Miura K, Yasuda R, Kawanabe H, Ogawa S, Eto Y. Nateglinide stimulates glucagon-like peptide-1 release by human intestinal L cells via a K(ATP) channel-independent mechanism. Biol Pharm Bull. 2011;34:671-6.

15. Tahara A, Matsuyama-Yokono A, Shibasaki M. Effects of antidiabetic drugs in high-fat diet and streptozotocin-nicotinamideinduced type 2 diabetic mice. Eur J Pharmacol. 2011;655:108-16.

16. Jung JA, Kaku K, Kim JH, Kim JR, Ko JW, Lee SY, et al. Additive postprandial glucose-lowering effects of mitiglinide and sitagliptin in patients with type 2 diabetes mellitus. Adv Ther. 2013;30:1018-29.

17. Stephens JW, Bodvarsdottir TB, Wareham K, Prior SL, Bracken RM, Lowe GD, et al. Effects of short-term therapy with glibenclamide and repaglinide on incretin hormones and oxidative damage associated with postprandial hyperglycaemia in people with type 2 diabetes mellitus. Diabetes Res Clin Pract. 2011;94:199-206.

18. Nauck MA, Heimesaat MM, Behle K, Holst JJ, Nauck MS, Ritzel R, et al. Effects of glucagon-like peptide 1 on counterregulatory hormone responses, cognitive functions, and insulin secretion during hyperinsulinemic, stepped hypoglycemic clamp experiments in healthy volunteers. J Clin Endocrinol Metab. 2002;87:1239-46.

19. Malin SK, Solomon TP, Blaszczak A, Finnegan S, Filion J, Kirwan JP. Pancreatic beta-cell function increases in a linear dose-response manner following exercise training in adults with prediabetes. Am J Physiol Endocrinol Metab. 2013;305:E1248-54.

20. McKillop AM, Duffy NA, Lindsay JR, Green BD, Patterson S, O'Harte FP, et al. Insulinotropic actions of nateglinide in type 2 diabetic patients and effects on dipeptidyl peptidase-IV activity and glucose-dependent insulinotropic polypeptide degradation. Eur J Endocrinol. 2009;161:877-85.

21. Holman RR, Haffner SM, McMurray JJ, Bethel MA, Holzhauer B, Hua TA, et al. Effect of nateglinide on the incidence of diabetes and cardiovascular events. N Engl J Med. 2010;362:1463-76.

22. DeFronzo RA, Okerson T, Viswanathan P, Guan X, Holcombe $\mathrm{JH}$, MacConell L. Effects of exenatide versus sitagliptin on postprandial glucose, insulin and glucagon secretion, gastric emptying, and caloric intake: a randomized, cross-over study. Curr Med Res Opin. 2008;24:2943-52. 